\title{
In Plane Optical Sensor Based on Organic Electronic Devices
}

\author{
Marc Koetse* ${ }^{* a}$, Peter Rensing ${ }^{\mathrm{b}}$, Gert van Heck ${ }^{\mathrm{a}}$, Ruben Sharpe ${ }^{\mathrm{a}}$, Bart Allard ${ }^{\mathrm{a}}$, Fokko Wieringa ${ }^{\mathrm{b}, \mathrm{c}}$, \\ Peter Kruijt $^{\mathrm{a}}$, Nicole Meulendijks ${ }^{\mathrm{a}}$, Henk Jansen ${ }^{\mathrm{b}}$ and Herman Schoo ${ }^{\mathrm{a}}$ \\ ${ }^{a}$ Holst Centre, PO Box 8550, $5605 \mathrm{KN}$ Eindhoven, The Netherlands \\ ${ }^{\mathrm{b}}$ TNO Science and Industry, P.O. Box 155, 2600 AD Delft, The Netherlands \\ ${ }^{\mathrm{c}}$ Erasmus Medical Centre, Department Biomedical Engineering, P.O. Box 2040, \\ 3000 CA, Rotterdam, The Netherlands
}

\begin{abstract}
Sensors based on organic electronic devices are emerging in a wide range of application areas. Here we present a sensor platform using organic light emitting diodes (OLED) and organic photodiodes (OPD) as active components. By means of lamination and interconnection technology the functional foils with OLED and OPD arrays form an in-plane optical sensor platform (IPOS). This platform can be extended with a wireless data and signal processing unit yielding a sensor node. The focus of our research is to engage the node in a healthcare application, in which a bandage is able to monitor the vital signs of a person, a so-called Smart Bandage. One of the principles that is described here is based on measuring the absorption modulation of blood volume induced by the pulse (photoplethysmography). The information from such a bandage could be used to monitor wound healing by measuring the perfusion in the skin. The OLED and OPD devices are manufactured on separate foils and glass substrates by means of printing and coating technologies. Furthermore, the modular approach allows for the application of the optical sensing unit in a variety of other fields including chemical sensing. This, ultimately enables the measurement of a large variety of physiological parameters using the same bandage and the same basic sensor architecture. Here we discuss the build-up of our device in general terms. Specific characteristics of the used OLEDs and OPDs are shown and finally we demonstrate the functionality by simultaneously recorded photoplethysmograms of our device and a clinical pulseoximeter.
\end{abstract}

Keywords: Organic photodiodes, OLED, Systems in foil, Photoplethysmography, Smart Bandage.

\section{INTRODUCTION}

In many application areas, including medical, life style, packaging, defense, and security, there is an increasing demand for cheap, flexible or wearable sensors. For example, in medium and high-care hospital departments vital patient parameters (e.g. heart beat frequency and arterial oxygen saturation $\left(\mathrm{SpO}_{2}\right)$ ) are monitored continuously. In many cases a disposable solution is preferred over reusable systems because of the need to maintain device sterility. In defense and security there is a demand for monitoring systems for both personnel (e.g. firemen, soldiers) and the environment (e.g. gasses, biological agents). Because of the demanding environments in which these applications are engaged, reliability, durability, wearability and wireless communication form serious requirements. The strict demands of such applications require reliable, yet cheap and disposable solutions. Large area electronics are very well suited, since they allow for low cost manufacturing. When using foils as substrate and organic electronic devices as the active components, cheap manufacturing methods such as coating and printing can be used.

The resulting conformal or even flexible devices are ideal for use in the above mentioned application areas, since they can be embedded in e.g. protecting garments The use of organic electronic devices in sensor applications is growing very rapidly. In recent years a number of examples have emerged at various research institutes and companies. Examples thereof include the use of organic photodiodes in a sensor array for x-ray imaging'; Oxygen and $\mathrm{pH}$ sensors ${ }^{2}$; Chemiluminescence sensors ${ }^{3}$; Photoluminescence sensors ${ }^{4}$; and chemical transmission sensors ${ }^{5,6}$.

*marc.koetse@tno.nl; www.holstcentre.com

Organic Field-Effect Transistors VII and Organic Semiconductors in Sensors and Bioelectronics, edited by Zhenan Bao, lain McCulloch, Ruth Shinar, George G. Malliaras,

Proc. of SPIE Vol. 7054, 70541I, (2008) - 0277-786X/08/\$18 - doi: 10.1117/12.794830

Proc. of SPIE Vol. $7054705411-1$ 
The key driver for the increasing research effort in this area is the promise for low cost, yet precise devices. It should be noted, that, in order to actually fulfill this promised low cost, the development of the technology to manaufacture these devices on an industrial scale is just as important as the development of the sensor platforms themselves.

Since many of the state of the art technologies in use today are derived from batch-wise processing, it will take quite an effort yet to come to reliable roll-to-roll processes that are absolutely needed to meet the cost targets. In fact, all processes that have so far been used for the fabrication of devices in the past, as well as new ones specific to foil substrates, will have have to be optimized or even completely newly developed.

\subsection{Organic in plane optical sensor for wound monitoring}

An in plane optical sensor (IPOS) can be seen as a generic platform for many application areas. It could, for example, allow for integration in credit card type sensor tags or textiles but can also be used for direct optical measurements on the skin. An interesting case is presented by the demand for a monitoring device for in situ measurement of the viability of skin grafts after reconstructive surgery of, for example, a burn wound. Current methodology involves hospitalization where, on a regular basis, the bandage is removed for visual inspection. In some cases more elaborate methods such as Laser Doppler Ultrasonography for measuring the perfusion in the graft may be used ${ }^{7}$. These diagnostic techniques, still require bandage removal, which by itself may disturb the wound curing process. The risk of infection or damage to the healing wound area is obviously not to be ignored. For this reason it would be advantageous to incorporate a monitoring device into the bandage. Such a device would allow to keep track of the healing process without unnecessary disturbances.

Organic electronic devices fabricated on foil combine a number of unique features that are in compliance with the requirements for such a monitoring device. These include low cost production by using roll-to-roll manufacturing; conformability or even flexibility that increases the comfort for the patient; and compatibility with modern wound care systems and dressings ${ }^{8}$. An artist impression of such a device, a smart bandage, is given in Figure 1. The fact that printing can be used as a processing technology allows for the construction of arrays of optical elements, which may be advantageous for large wound areas.

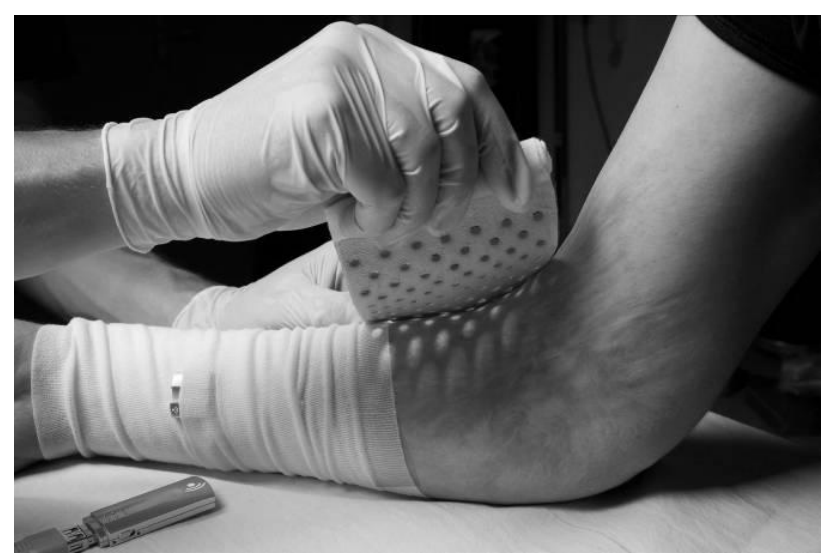

Fig. 1. Artist impression of a smart bandage, integrating organic electronic devices with a wound dressing.

In the present paper an approach is described to develop a foil based sensor, capable of measuring the perfusion of the microvascular tissue in the wound area by means of photoplethysmography ${ }^{9}$ (PPG). The nowadays widely applied pulseoximeter uses PPGs to measure heart beat frequency and the arterial level of blood oxygen saturation $\left(\mathrm{SpO}_{2}\right)$. This is achieved by measuring the change in absorption due to the pulse (blood volume) at two wavelengths, typically in the red and near infrared. For monitoring relative changes in perfusion only one wavelength is sufficient, which simplifies the design and the manufacture of the sensor device. Obviously, it is very well possible to combine several wavelengths on one foil to monitor more parameters. In this proceedings we discuss some relevant characteristics of the OLEDs, OPDs and the combination of the two for the actual measurement of a PPG. 


\section{APPROACH}

Apart from being a platform for application in many different areas, the IPOS may also be seen as a platform for the development and testing of manufacturing technologies. These include printing technologies for the active materials, barrier development for encapsulation of the devices, micro-precision lamination and interconnection technologies for the final device assembly, printing of conductive structures, and lithography on foil.

To demonstrate the possibilities of this technology we aim to create an array of organic photo detectors (OPD) and a compatible array of organic light emitting diodes (OLED). The OPDs are based on a blend of poly(3-hexylthiophene) (P3HT, Merck Chemicals Ltd) and [6,6]-phenyl C61butyric acid methyl ester (PCBM, Solenne BV). Being the work horse for photovoltaic research, this blend has also been well studied for use in detectors ${ }^{10,11}$. This blend has an optical band gap of $650 \mathrm{~nm}$ which is sufficient for the detection of part of the red light.

The light emitting polymers (LEP) used for this study are a red emitting ${ }^{12}$ and yellow emitting ${ }^{13}$ material; both obtained from Merck KGaG. The red emitting material $\left(\lambda_{\max } 670 \mathrm{~nm}\right)$ shows a large overlap with the absorption spectrum of the photodiode blend(fig 5). Also the emission overlaps with the prime wavelength for the PPG measurement, $650 \mathrm{~nm}$. This makes this material particularly useful for the sensor (fig 2). The emission of the yellow material $\left(\lambda_{\max } 575 \mathrm{~nm}\right)$ has a strong emission shoulder in the red spectral region and has also a strong overlap with the diode spectrum.

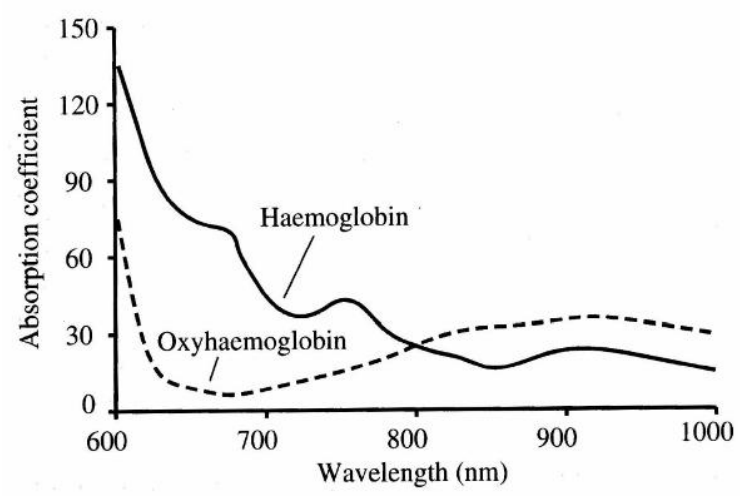

Fig. 2. Optical absorption of Haemoglobin and Oxy-haemoglobin in the spectral region used for clinical pulse-oximetry. This region is often called the "near infrared window" because photons can penetrate deeply into tissue. Below $600 \mathrm{~nm}$ the absorption of light by notably melanin becomes too strong and beyond $1000 \mathrm{~nm}$ the water absorption rises quickly. ${ }^{9}$

In order to maximize the degrees of freedom for design and manufacturing of the devices, we decided to fabricate the detectors and LEDs on separate foils. For example, this allows comparison of various printing methods and coating technologies on a wide range of substrates. In this study we made use of poly(ethylenenaphthalene) (PEN) and glass. A further important benefit of this approach is that we are essentially free to choose the order of functional foils in the final device, which allows easy variation of the device's architecture.

For the device discussed in this study we opted for a three foil assembly containing an OLED foil with an OPD foil laminated on top. The device is complemented with a flexible circuit board containing noise filters (band pass $0.5-17$ Hz), logarithmic amplifier (AD8304, Analog Devices) and a DC/DC converter (Max1896, Maxim) for the power supply of the OLEDs. Data collection and OLED driving is controlled with a microprocessor embedded on a multifunctional wireless node ${ }^{14}$. The interconnection between the foils is achieved, using a proprietary lamination and interconnection technology. Figure 3 shows a mock up version that is used for testing of the interconnection technology and of the attached node as well as a picture of the OLED and OPD foils.

The functional foils were processed in a batch-wise manner. A typical work flow for both the OLED and OPD foils involves:

- Lamination of a substrate to a carrier;

- Deposition of the barrier; ${ }^{15}$ 
- Deposition and patterning of the anode and shunt lines;

- Deposition of PEDOT:PSS and the active layer by means of spin coating or inkjet printing;

- Evaporation of the cathode;

- Thin film encapsulation. ${ }^{15}$

The first two steps are omitted for processing on glass substrates. The resulting foil devices are depicted in Fig. 3.

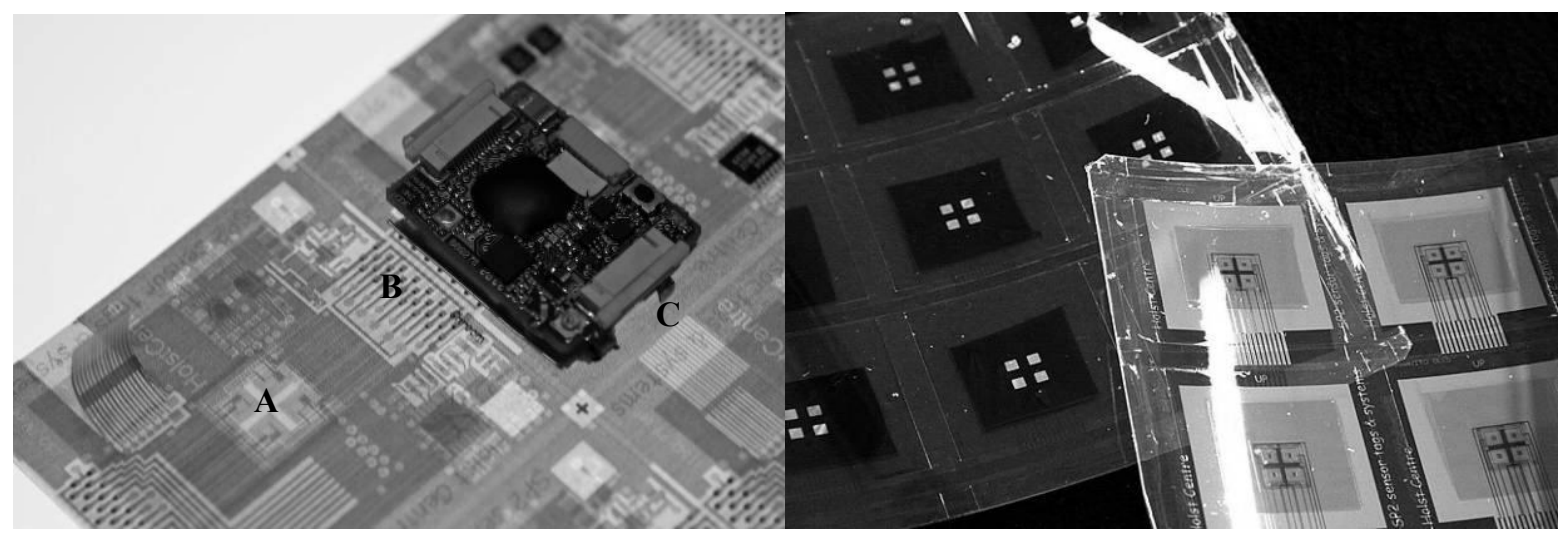

Fig. 3. Left: Mock up version of sensor node foil (stack of 3 foil layers). This uncut foil stack is printed with several devices in a repetitive pattern. The optical array (A) and interconnection (B) can be seen on a device where a wireless node is not yet placed. Note the upward curved section of the top interconnection layer (left of A) which serves as a flexfoil connection to the wireless node. The neighboring device is equipped with a wireless node (C). Right Uncut foils containing several OLED (foreground) and OPD (background) devices.

Both foils can be made either bottom or top emissive (receptive). Although processing on glass is a well know procedure, our devices were designed to be double sided. In such a device light leakage through the substrate can be avoided by using a top emissive OLED ${ }^{16}$ on one side of the substrate and a bottom receptive OPD on the other. This light will be emitted nearly directly into the skin and only reflected and scattered light will pass through a single substrate. Classical encapsulation with a metal lid is not useful because of both transparency and flexibility issues and therefore a transparent thin film encapsulation was used. A further advantage of using double sided devices is that spincoating can be used without the risk of cross-contamination of the devices. A schematic overview of the architecture of both device designs is given in figure 4. The photodiodes with an area of $1 \mathrm{~mm}^{2}$ are placed behind the LEDs (area 8 $\mathrm{mm}^{2}$ ) and receive the reflected light via an opening in the middle.

A

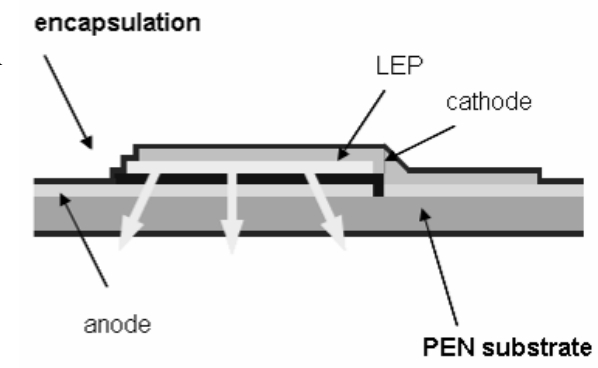

B

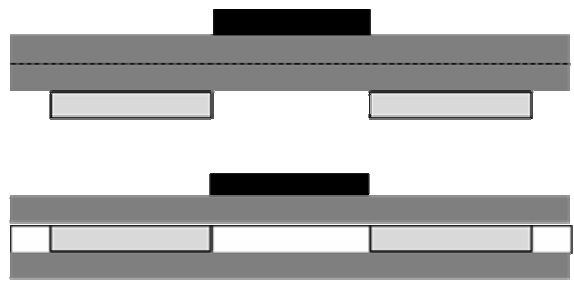

Fig. 4. Detailed cross section of an oled structure (A) and schematic cross section (B,C) of different foil stack configuration. B shows the architecture in case a top emissive OLED (light gray) used in combination with a bottom receptive OPD (black). The dotted line may be seen as the adhesive in case foil is the substrate. C depicts the combination of both bottom emitting and receptive devices. For clarity, the dimensions of the actual are strongly exaggerated. 


\section{RESULTS AND DISCUSSION}

\subsection{Top-emissive OLEDs}

Bottom-emissive OLEDs are considered Lambertian emitters. As indicated before this might reduce the amount of light that is able to penetrate the skin because of "leakage" into the substrate. Clearly, the loss of light is strongly dependent on the thickness of the substrate. For the devices on glass and the first generation on foil (Fig. 4, B) we therefore chose to use top emissive OLEDs. Here the light only has to pass trough the thin film encapsulation only $(<20 \mu \mathrm{m})$, minimizing this loss.

The red emitting material shows a peak emission at $670 \mathrm{~nm}$ and has a large overlap with the absorption spectrum of the photodiode blend (fig 5). Also the emission overlaps with the primary wavelength for the PPG measurement, which is around $650 \mathrm{~nm}$. This makes this material particularly useful for the sensor (Fig 2).

The bottom-emissive devices had a maximum efficiency of $1.5 \mathrm{Cd} / \mathrm{A}$. In top emissive devices this was reduced to a maximum efficiency of $0.45 \mathrm{Cd} / \mathrm{A}$ at $8 \mathrm{~V}$ and $320 \mathrm{Cd} / \mathrm{m}^{2}$ as measured with a luminance meter. The area of the LED was 8 $\mathrm{mm}^{2}$, implying a current of $5.6 \mathrm{~mA}$. These results show a drawback of top emissive devices, namely that their luminescence is generally much lower than the luminescence of their bottom emissive counterpart. This is partly due to the reduced transparency of the cathode $(60-70 \%)$ and partly due to the angle dependant emission, caused by a cavity effect. Figure 5 shows a conoscopic measurement (Eldim, EZ-Contrast L160D) of a top-emissive device driven at 9 V. Clearly the distribution of the emitted light is not Lambertian but has a maximum between $40^{\circ}$ and $50^{\circ}$. We do not expect this to cause problems in the final PPG measurements since these depend mainly on back scattered light and the design with 4 photodiodes allows for a relatively large measurement area.

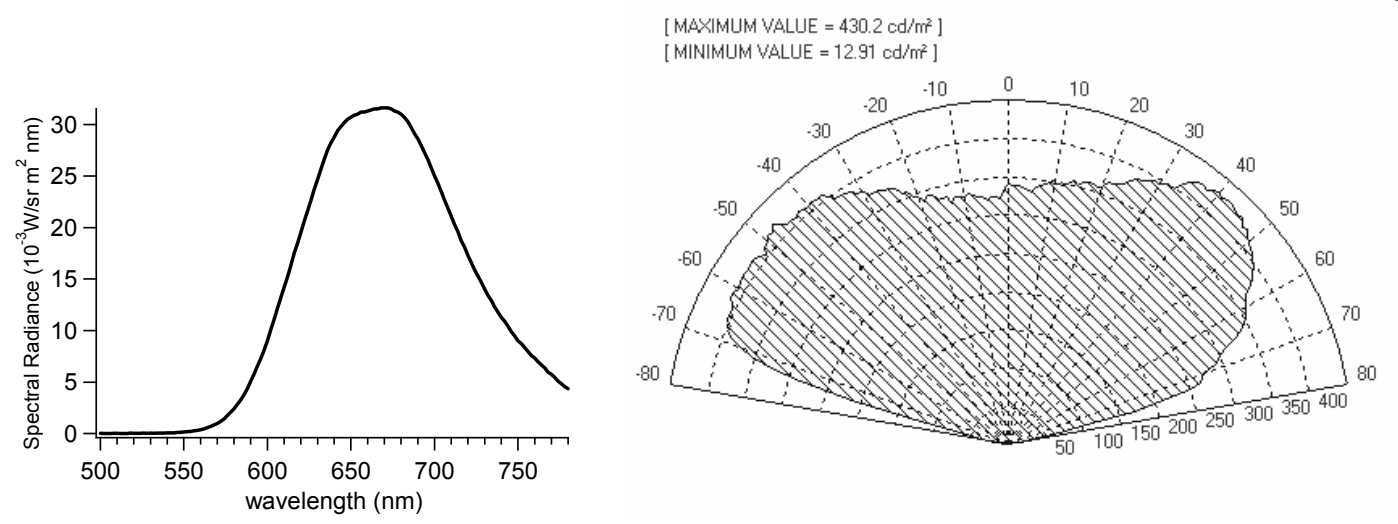

Fig 5 Emission spectrum of the red emitting OLED (left) and Cross section of a conoscopic image in Luminance $\left(\mathrm{Cd} / \mathrm{m}^{2}\right) \mathrm{vs}$. the viewing angle.

\subsection{Organic photodiodes}

The photodiodes used in this study were bottom-receptive and had an area of $1 \mathrm{~mm}^{2}$. The active layer was spin coated from a chlorine free solvent. No separate thermal annealing was required. A TEM picture (Fig. 6) of an inkjet printed layer, using the same solvent, shows similar features as reported in literature. ${ }^{17}$ This indicates that the morphology is probably very similar to those found in films obtained by chlorinated solvents such as o-dichlorobenzene (ODCB). This was corroborated with IV-measurements of both spin coated and inkjet printed devices using LiF/Al as cathode. Both showed high shortcut currents (Jsc) of $10 \mathrm{~mA} / \mathrm{cm}^{2}$ and $8.7 \mathrm{~mA} / \mathrm{cm}^{2}$, respectively (see Fig. 6.) under approximately 100 $\mathrm{mW} / \mathrm{cm}^{2}$ white light illumination. The open circuit voltage (Voc) was $0.58 \mathrm{~V}$ with a fill factor (FF) of 0.56 for the spin coated device. The printed device showed a slightly lower Voc of $0.54 \mathrm{~V}$ but a dramatically lower FF. We attribute this to the inhomogeneity of the printed layer. Optimization of the printing process, including ink formulation, is currently being carried out. It should be noted that these measurements were not performed under standard (AM1.5) conditions. The results give therefore only an indication of the actual performance of the devices, but can be used to compare the devices presented here. 

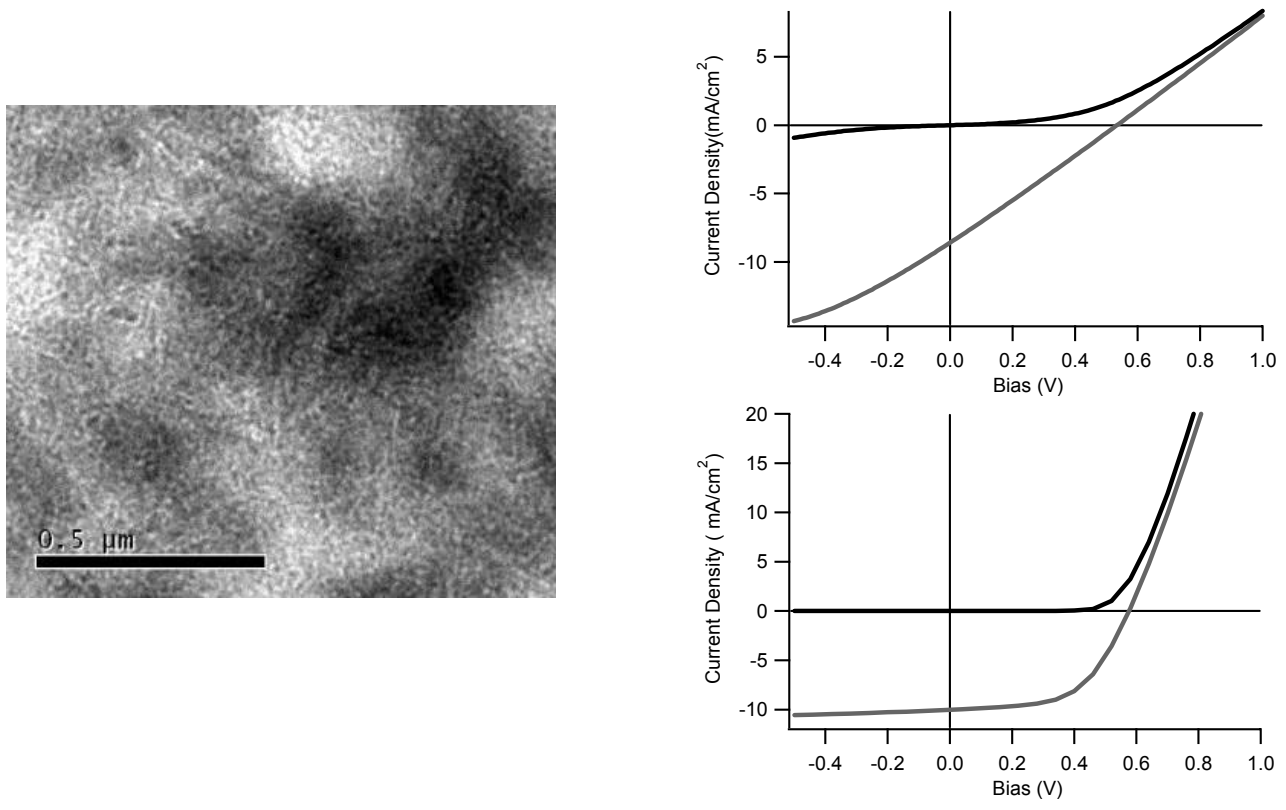

Fig. 6. TEM image of an inkjet printed film of P3HT/PCBM blend (left) and IV curves of a spin coated device (bottom right) and inkjet printed device (top right). The dark curves are given in black, the curves measured under illumination in gray.

The noise levels of our devices can be estimated with relative ease from the shunt resistance $\left(\mathrm{R}_{\mathrm{sh}}\right)$ measured in the dark. For the $1 \mathrm{~mm}^{2}$ spin coated devices we found a typical value for $R_{\text {sh }}$ of $10 \mathrm{M} \Omega$. From this the thermal current noise density $\left(\mathrm{I}_{\mathrm{tn}}\right)$ can be calculated using $\mathrm{I}_{\mathrm{tn}}=\sqrt{ }\left(4 \mathrm{kT} / \mathrm{R}_{\mathrm{sh}}\right)=40 \mathrm{fA} / \mathrm{Hz}^{1 / 2}$. The measured current noise density was found to be $70 \mathrm{fA} / \mathrm{Hz}^{1 / 2}$. This indicates that the origin of noise in the devices is not purely determined by $\mathrm{R}_{\mathrm{sh}}$. The derived Noise Equivalent Power (NEP) was found to be $2.8 \times 10^{-13} \mathrm{~W} / \mathrm{Hz}^{1 / 2}$, based on an estimated spectral response of $0.25 \mathrm{~A} / \mathrm{W}^{11}$. This is an order of magnitude higher than reported for optimized organic photodiodes in literature. ${ }^{11}$ Although not yet optimal, we feel confident to use the spin coated devices in the sensor, since the currents to be measured are in the order of $\mu \mathrm{A}$, with an AC component of tens nA. which is well above the measured current noise density. The inkjet printed devices have a too low $\mathrm{R}_{\text {sh }}$ yet to be used in the, here described, sensor platform.

\subsection{Photoplethysmography with OLEDs and OPDs}

As a proof-of-principle we made use of a double sided, glass based device using two red emitting OLEDs and one photodiode. The OLEDs were driven at 9V. The measured photocurrents were filtered (band pass $0.5-17 \mathrm{~Hz}$ ) and the AC component was amplified using a logarithmic amplifier (AD8304). The measurement was performed on the right index finger of a test person. A simultaneous measurement with a commercial pulseoximeter (Nelcor N200) on the middle finger served as control. Both resulting photoplethysmograms are shown in Fig. 7.

The signal of the organic device and the clinical pulseoximeter match perfectly thus showing that our sensor can well be used for measuring the pulse. The PPG waveform is typical for a measurement on a finger. ${ }^{9}$ As a result of postprocessing, the commercial device delivers a strongly smoothed signal, whereas the signal of the organic device was only slightly smoothed electronically. The total signal measured by the photodiode was $10 \mu \mathrm{A}$, the relevant modulation (AC signal) was approximately $50 \mathrm{nA}$, estimated from the amplifier characteristics. ${ }^{17}$ 


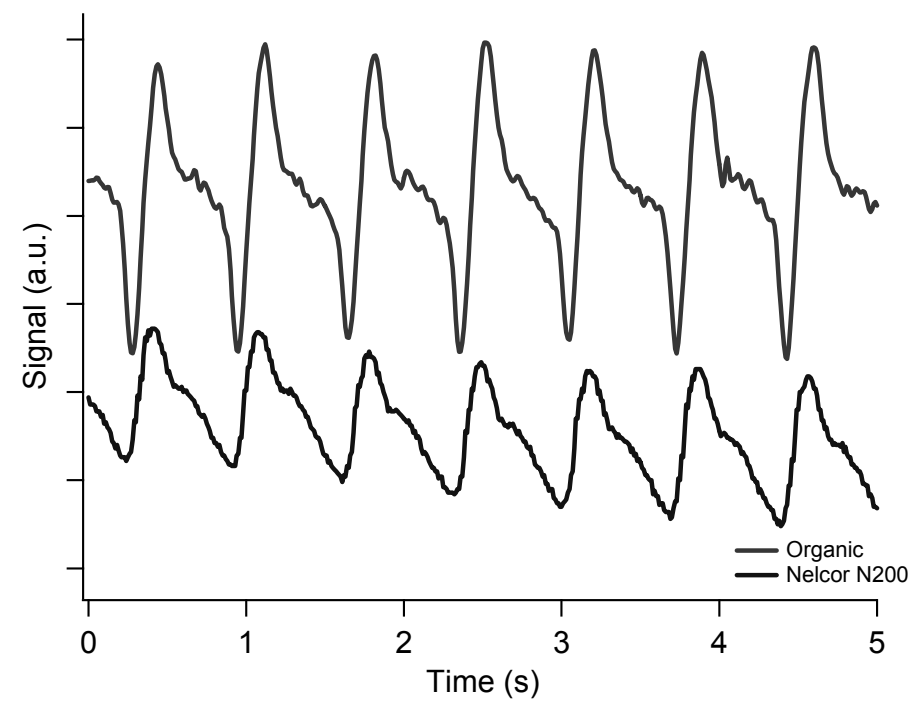

Fig. 7. Photoplethysmogram showing the pulse of a test person measured with the organic device (top) and a commercial pulse-oximeter (bottom). The signals have been shifted on the y axis for clarity.

A serious drawback of the use of the low efficiency OLEDs is the heat dissipation of the device. The temperature of the device may rapidly exceed $60{ }^{\circ} \mathrm{C}$, which makes long term skin contact impossible. To overcome this we performed similar measurements using yellow emitting OLEDs. The improved efficiency $(10 \mathrm{Cd} / \mathrm{A}$ versus $0.45 \mathrm{Cd} / \mathrm{A}$ for the red emitting devices) allowed driving the devices at much lower voltage and current density, hence decreasing the operating temperature to below $35^{\circ} \mathrm{C}$. The obtained PPG was comparable to that measured before, although the current of the AC signal was reduced to $10 \mathrm{nA}$. Apparently there is enough emission in the red part of the spectrum to yield viable measurements. Other skin areas were also tried including hand, wrist and forehead. On all locations a PPG could be measured, the signal being the strongest on the head and fingers, which can be expected since in these areas the capillaries lie closest to the skin surface.

\section{CONCLUSIONS}

Sensors based on organic electronic devices receive increasing attention from academia, research institutes and industry. Products, mainly for point of care applications, are now slowly entering the market. The platform discussed in this paper allows for off-line point of care applications but can also be used for direct measurements of physiological processes on or in the body. More specifically the platform was used for photoplethysmography, measuring the skin perfusion using the modulation of the capillary volume in the skin. It contains an in-plane optical sensor node containing OLEDs and OPDs on foil that can be integrated with existing electronics using lamination technologies.

For testing purposes the device was designed such that it could be manufactured either on glass (double sided) or on foil. This implied the use of top emitting OLEDs and bottom receptive OPDs. The applied red emitting device had a relatively low efficiency of $0.45 \mathrm{Cd} / \mathrm{A}$ and also showed a strong angle dependent emission. The strongest luminance was measured between $40^{\circ}$ and $50^{\circ}$.

The OPDs were manufactured with chlorine free solvents. The resulting layers showed a morphology comparable to that reported for layers processed from more common solvents such as ODCB. Good quality devices with high shortcut current densities $\left(10 \mathrm{~mA} / \mathrm{cm}^{2}\right)$ could be made using spin coating. Inkjet printed devices were of lower quality, mainly due to the inhomogeneity of the layers, reflected in the low fill factor and shunt resistance. The short circuit current, however, was almost equal to that of spin coated devices. Preliminary noise measurements indicate an order of magnitude higher Noise Equivalent Power than reported in literature, but still well below the expected measurement window. 
With the combination of these OLEDs and OPDs a photoplethysmogram was measured on the finger of a test person. The signal matched a simultaneously measured PPG from a commercial pulseoximeter. The low efficiency red emitting OLED produced an unacceptable amount of heat, which could be remedied by using a higher efficiency yellow emitting material. This proves that organic optoelectronic devices can well be used for the direct measurement of relative changes in physiological parameters such as the skin perfusion in.

\section{REFERENCES}

[1] Ng T. N., Wong W.S., Chabinyc M. L., Sambandian S.,. Street R. A., "Flexible image sensor array with bulk heterojunction organic photodiode," Appl. Phys. Lett, 92, 213303 (2008).

[2] Kraker E., Haasse A., Lamprecht B., Jacopic G., Konrad C., Köstler S., "Integrated organic electronic based optochemical sensors using polarization filters," Appl. Phys. Lett. 92, 033302 (2008).

[3] Hofmann O., Milller P., Sullivan P., Jones T.S., deMello J. C., Bradley D. D.C., deMello A. J., "Thin-film organic photodiodes as integrated detectors for microscale chemiluminescence arrays," Sensors and Actuators B, 1006, 878884 (2005).

[4] Pieler R., Füreder, E., Sonnleitner, M., "Printed photonics for lab-on-chip applications," Proc. SPIE 6739, 673919 (2007).

[5] Sharpe, R., Rensing P., Van Heck G., Allard, B., Koetse M., Meulendijks N., Kruijt P., Tijdink M., De Zwart, R., Van Veen, S., Schoo, H. "Polymer-based micro-array sensors," Proc. Spie 6659, 665908 (2007),

[6] Schoo, H., Van Veen, S., Bekman, H., "Sensor comprising polymeric components", Int. Pat. Appl., WO2005015173.

[7] Yuen, J., “Techniques of External Monitoring of Buried Free Flaps” Annals of Plastic Surgery. 55 460-465 (2005).

[8] Fonder M., Lazarus G, Cowan D., Aronson-Cook B., Kohli A., Mamelak A., "Treating the chronic wound: A practical approach to the care of nonhealing wounds and wound care dressings," Dan. Med. Bull. 55, 72-77 (2008).

[9] Allen J., "Photoplethysmography and its application in clinical physiological measurement," Physiol. Meas., 28 R1R39 (2007).

[10] Schilinsky P., Waldrauf C., Hauch J., Brabec C., "Polymer photovoltaic detectors: Progress and recent developments," Thin Solid Films, 451-452, 105-108 (2004).

[11] Ramuz M., Bürgi L., Winnewisser C., Seitz P., "High sensitivity organic photodiodes with low dark currents and increase lifetimes," Org. Elect. 9, 369-376 (2008).

[12] Falcou A., Becker H., Breuning E., Buesing A., Heun S., Parham A., Spreitzer H., Steiger J., Stoessel P., "Light Emitting Polymer Materials: The working base for flexible full color displays," Proceedings of the third International Meeting on Information Display, 1053-1056 (2003).

[13] Becker H., Spreitzer H., Kreuder W., Kluge E., Schenk H., Parker I., Cao Y., "Soluble PPVs with enhanced performance, a mechanistic approach," Adv. Mater. 12, 42-48 (2000).

[14] Gyselinckx B., Penders J., Vullers R., "Potential and challenges of body area networks for cardiac monitoring," J. Electrocardiol., 40 S165-S168 (2007).

[15] Hemerik M., Van Erven R., Vangheluwe R., Yang J., Van Rijswijk T., Winters R., Van Rens B., "Lifetime of ThinFilm Encapsulation and its Impact on OLED Device Performance," SID Symposium Digest 37, 1571 (2006)

[16] Riel H., Karg S., Beierlein T., Ruhstaller B., Rieß W., "Phosphoressent top emitting organic light-emitting devices with improved light outcoupling," Appl. Phys. Lett. 82, 466 (2003).

[17] Yang X., Loos J., Veenstra S., Verhees W., Wienk M., Kroon J., Michels M., Janssen R., "Nanoscale morphology of high-Performance polymer solar cells," Nano Lett.,5 , 579 -583 (2005).

[18] Analogue Devices AD8304, http://www.analog.com/static/imported-files/data_sheets/AD8304.pdf 